\title{
Bone marrow mesenchymal stem cells overexpressing human basic fibroblast growth factor increase vasculogenesis in ischemic rats
}

\author{
J.C. Zhang ${ }^{1}$, G.F. Zheng ${ }^{2}$, L. Wu ${ }^{1}$, L.Y. Ou Yang ${ }^{1}$ and W.X. $\mathrm{Li}^{1}$ \\ ${ }^{1}$ Department of Vascular Surgery, The First Affiliated Hospital of Fujian Medical University, Fuzhou, China \\ ${ }^{2}$ Department of Vascular Surgery, The People's Hospital of Ganzhou, Ganzhou, China
}

\begin{abstract}
Administration or expression of growth factors, as well as implantation of autologous bone marrow cells, promote in vivo angiogenesis. This study investigated the angiogenic potential of combining both approaches through the allogenic transplantation of bone marrow-derived mesenchymal stem cells (MSCs) expressing human basic fibroblast growth factor (hbFGF). After establishing a hind limb ischemia model in Sprague Dawley rats, the animals were randomly divided into four treatment groups: MSCs expressing green fluorescent protein (GFP-MSC), MSCs expressing hbFGF (hbFGF-MSC), MSC controls, and phosphate-buffered saline (PBS) controls. After 2 weeks, MSC survival and differentiation, hbFGF and vascular endothelial growth factor (VEGF) expression, and microvessel density of ischemic muscles were determined. Stable hbFGF expression was observed in the hbFGF-MSC group after 2 weeks. More hbFGF-MSCs than GFP-MSCs survived and differentiated into vascular endothelial cells $(\mathrm{P}<0.001)$; however, their differentiation rates were similar. Moreover, allogenic transplantation of hbFGF-MSCs increased VEGF expression $(P=0.008)$ and microvessel density $(P<0.001)$. Transplantation of hbFGF-expressing MSCs promoted angiogenesis in an in vivo hind limb ischemia model by increasing the survival of transplanted cells that subsequently differentiated into vascular endothelial cells. This study showed the therapeutic potential of combining cell-based therapy with gene therapy to treat ischemic disease.
\end{abstract}

Key words: Angiogenesis; Basic fibroblast growth factor; Bone marrow mesenchymal stem cells; Gene transfection; Ischemia

\section{Introduction}

Increasing in vivo angiogenesis is a therapeutic strategy for treating ischemic diseases, including peripheral artery disease, heart disease, myocardial infarction and stroke. Administration of angiogenic factors, such as vascular endothelial growth factor (VEGF), human basic fibroblast growth factor (hbFGF), and hepatocyte growth factor (HGF) (1-3), or gene transfer of these growth factors (4) have been reported. Intramuscular transfer of both the VEGF165 and HGF genes increased perfusion and decreased necrosis in ischemic mouse hind limbs via neovascularization (5). Similar results were obtained with AGGF1 (angiogenic factor with $G$ patch and FHA domains 1 ), a relatively newly isolated angiogenic factor that significantly reduced the ambulatory impairment associated with limb ischemia (6).

In addition to direct or systemic administration of growth factors to promote angiogenesis, implantation of autologous bone marrow cells promoted angiogenesis in a rat ischemic hind limb model $(7,8)$. Specifically, injection of purified bone marrow cells (BMCs) that have the potential to differentiate into endothelial cells (9) and produce angiogenic growth factors (10) increased blood flow in ischemic hind limbs 4 weeks after transplantation (7). Furthermore, increased exercise capacity was observed after implantation of autologous BMCs in a rat ischemic hind limb model (8). Similar functional recovery has been observed with allogenic transfer of mesenchymal stem cells (MSCs) derived from bone marrow or adipose tissue in an in vivo model of ischemic stroke (11). Although stem cell transplantation has been used to stimulate vasculogenesis with favorable efficacy, the reparative capacity of MSCs decline with age, which may be enhanced by lentiviral-mediated expression of myocardin and telomerase reverse transcriptase (12) or

Correspondence: J.C. Zhang, Department of Vascular Surgery, Institute of Vascular and Endovascular Surgery, The First Affiliated Hospital of Fujian Medical University, No. 20, ChaZhong Road, TaiJiang District, Fuzhou 35005, China. Fax: +86-591-8335-6180. E-mail: fjfyzjc@163.com 
culture expansion in media supplemented with growth factors prior to transplantation (13). Moreover, stem cell survival after transplantation remains low, negatively impacting their therapeutic efficacy.

Because bFGF has the potential to promote angiogenesis $(14,15)$ and also increase the in vivo survival and proliferation of stem cells (16), this study aimed to test the hypothesis that bone marrow-derived MSCs overexpressing hbFGF would increase angiogenesis to a greater extent than MSCs alone. To test this hypothesis, rats with hind limb ischemia were treated with MSCs expressing green fluorescent protein (GFP-MSC group), MSCs expressing hbFGF (hbFGF-MSC group), MSCs alone (MSC group), or phosphate-buffered saline (PBS control group). After 2 weeks, MSC survival was determined by GFP fluorescence, and differentiation was determined by performing immunofluorescence assays of CD31 expression. hbFGF and VEGF expression in gastrocnemius muscle was determined by Western blot analyses. In addition, microvessel density (MVD) was determined by immunohistochemical analysis of von Willebrand factor (VWF) expression. Our results may form the basis for further clinical studies assessing the therapeutic efficacy of hbFGF overexpression in MSCs prior to autologous transplantation.

\section{Material and Methods}

\section{Animals}

Thirty healthy 6-week-old male Sprague Dawley rats were purchased from the Shanghai SLAC Laboratory Animal Co., Ltd. (China). Animals were acclimated in the Fujian Medical University Animal Center for 1 week with free access to food and water. The animals were fasted for $12 \mathrm{~h}$ prior to the beginning of the experiments. The study was approved by the Institutional Review Board of The First Affiliated Hospital of Fujian Medical University.

\section{MSC isolation}

The isolation and identification of MSCs were performed as we previously described (17). Briefly, 6 Sprague Dawley rats were euthanized by cervical dislocation, and bilateral femurs and tibias were collected. After the metaphysis was removed, the exposed bone marrow cavity was flushed with Dulbecco's modified Eagle medium (DMEM; Hyclone, USA) containing $10 \%$ fetal bovine serum (FBS, Hyclone). The fluid was harvested and centrifuged at $180 \mathrm{~g}$ for $10 \mathrm{~min}$. After the supernatant was removed, the cells were resuspended in culture medium and lymphocyte separation medium at a ratio of 2:1 (v:v). The cell suspension was added to $1.077 \mathrm{~g} /$ L lymphocyte separation medium ( $d=1.077 \mathrm{~g} / \mathrm{mL}, \mathrm{H} \& Y$ Bio, China) followed by centrifugation at $360 \mathrm{~g}$ for $15 \mathrm{~min}$. The white, cloudy cell layer was collected, and the cells were washed twice with L-DMEM. Single-cell suspensions were prepared with L-DMEM containing 10\% FBS. Cells at $1 \times 10^{6}$ cells $/ \mathrm{cm}^{2}$ were seeded into $25-\mathrm{cm}$ flasks and maintained in an incubator at $37^{\circ} \mathrm{C}$ in a humidified environment with $5 \% \mathrm{CO}_{2}$.

\section{MSC surface antigen expression assayed by flow cytometry}

MSCs were observed with an inverted microscope (Olympus, Japan). Cells of the third passage (P3) were used for all subsequent experiments as previously described (18). Cells were harvested at $80-90 \%$ confluency by digestion with $0.125 \%$ trypsin. Cell suspensions containing $2 \times 10^{6}$ cells were transferred into $101.5-\mathrm{mL}$ Eppendorf tubes and then incubated with $10 \mu \mathrm{L}$ of the following mouse anti-rat monoclonal antibodies: CD90-R-phycoerythrin $(\mathrm{PE})$, CD34-fluorescein isothiocyanate (FITC), CD44-PE, CD11b/c-FITC or isotype controls (all from Beckman Coulter, USA) for $15 \mathrm{~min}$ in the dark at $37^{\circ} \mathrm{C}$. Cells were washed twice with $1 \mathrm{~mL}$ PBS followed by centrifugation at $360 \mathrm{~g}$ for $5 \mathrm{~min}$. After resuspension at $0.5 \times 10^{6} \mathrm{cells} / \mathrm{mL}$ in PBS, surface antigens were detected by flow cytometry (Beckman Coulter); the System II software (Beckman Coulter) was used for data acquisition and analysis. MSCs were characterized as negative for CD11b and CD34 hematopoietic cell marker expression (19) and positive for CD44 and CD90 MSC marker expression (20).

\section{Preparation of hbFGF-expressing lentivirus}

Briefly, human cDNA was amplified by polymerase chain reaction (PCR) using the following $h b F G F$-specific primers (the Agel restriction site is underlined) from Shanghai Genechem Co., Ltd. (China): sense: 5'-CATGGGCTGGAC GAGGAATGGCAGCCGGGAGCATC-3' and antisense: 5'TCACCATGGTGGCGACCGGTACGTAGCTCTTAGCAG ACATTGGAAG-3'.

The PCR conditions were as follows: denaturation at $94^{\circ} \mathrm{C}$ for $30 \mathrm{~s} ; 30$ cycles of $94^{\circ} \mathrm{C}$ for $30 \mathrm{~s}, 55^{\circ} \mathrm{C}$ for $30 \mathrm{~s}$, $72^{\circ} \mathrm{C}$ for $30 \mathrm{~s}$, and a final extension at $72^{\circ} \mathrm{C}$ for $10 \mathrm{~min}$. After the PCR product was subjected to $1.1 \%$ agarose gel electrophoresis and purified using a plasmid extraction kit (Qiagen, The Netherlands), it was inserted into the pGC FU vector (Shanghai Genechem Co., Ltd.) at the Agel site. pGC FU-hbFGF vectors were identified by PCR followed by DNA sequencing to confirm the insertion of the target gene.

The pGC FU-hbFGF vector, pHelper 1.0 vector, and pHelper 2.0 vector (all from Shanghai Genechem Co., Ltd.) were transfected into 293T cells (Shanghai Genechem Co., Ltd.) in the presence of Lipofectamine 2000 (Invitrogen, USA). After 2 days, the cell culture supernatant was collected and repeatedly centrifuged at $4000 \mathrm{~g}$ for $10 \mathrm{~min}$. After the lentivirus was concentrated by filtration, it was stored at $-80^{\circ} \mathrm{C}$. Real-time quantitative PCR was performed to determine the lentivirus titer.

\section{Lentiviral-mediated MSC transduction}

P3 MSCs with good growth were used to prepare single-cell suspensions, which were then seeded onto 6 well plates at a density of $1 \times 10^{4}$ cells $/ \mathrm{cm}^{2}$. After 1 day, 
$1 \mathrm{~mL}$ fresh medium was added. When the cell confluency reached $30 \%$, cells were infected with GFP- or hbFGFexpressing recombinant virus at a multiplicity of infection of 50 as previously described (21). The hbFGF-expressing lentiviruses also expressed GFP. After $10 \mathrm{~h}$, the medium was removed, and cells were washed with PBS followed by addition of fresh medium. Cells were passaged using routine methods and observed daily by fluorescence microscopy to confirm continued GFP expression.

\section{Establishment of an in vivo ischemia model and MSC transplantation}

Rats were fasted for $12 \mathrm{~h}$ before surgery but were given ad libitum access to water. Animals were intraperitoneally anesthetized with $10 \%$ chloral hydrate $(3 \mathrm{~mL} / \mathrm{kg})$. Under aseptic conditions, a longitudinal incision was made in the hind limb 2-3 cm from the femoral artery. After the femoral artery was ligated at the level of the inguinal ligament, it and its branches were removed while protecting the femoral nerve.

At $24 \mathrm{~h}$ post-induction of hind limb ischemia, the rats were randomly divided into four groups ( $n=6 /$ group): control, MSC, GFP-MSC, and hbFGF-MSC. Animals in the MSC, GFP-MSC, and hbFGF-MSC groups received injections of $5 \times 10^{6}$ cells in $0.5 \mathrm{~mL}$ PBS into the adductors, tibialis anterior, and gastrocnemius muscles of the ischemic limb. Animals in the control group received an injection of $0.5 \mathrm{~mL}$ PBS into the ischemic limb.

No difference in tissue necrosis was observed between the hbFGF-MSC and control groups. Specifically, after induction of hind limb ischemia, ischemic necrosis was found in one or two toes of 3 animals in the PBS group; no necrosis was observed in the other groups. In addition, wound infection and delayed wound healing were found in 2 rats in the PBS control group. However, all animals survived. Apparent ambulatory impairment was observed within 3-5 days after surgery; however, normal activity was observed 1 week after surgery.

At 2 weeks post-transplantation, which was considered a chronic condition, blood was collected from the abdominal aorta, after which the animals were sacrificed and the adductor muscle and gastrocnemius were collected. A fraction of skeletal muscle was used for histological examination, and the remaining tissues were stored at $-80^{\circ} \mathrm{C}$ for later use.

\section{Immunohistochemistry and immunocytochemical analyses}

Muscle cross-sections ( $8 \mu \mathrm{m}$ ) were prepared for immunohistochemistry using the SP-9000 two-step method (Beijing Zhongshan Biotech, China). Primary antibodies specific for vWF (Cat No. sc-53466; Santa Cruz Biotechnology, USA) and CD31 (Cat No. sc-13537, Santa Cruz Biotechnology), goat anti-rabbit IgG secondary antibodies (Beijing Zhongshan Biotech) and a diaminobenzidine (DAB) substrate kit (Beijing Zhongshan Biotech) were used. vWFpositive cells appeared brownish-yellow, and the number of positive cells (i.e., microvessel number) was counted at a high magnification of $200 \times$ (excluding the tubes formed by more than three positive cells). CD31-positive cells were identified by their red fluorescence using a confocal laserscanning microscope (ZEISS LSM510, Germany).

\section{Western blot analysis}

Muscles were cut into pieces and homogenized mechanically for $10 \mathrm{~min}$ (170 rpm). After determination of protein concentration, $100 \mu \mathrm{g}$ protein was separated by sodium dodecyl sulfate polyacrylamide gel electrophoresis (SDS-PAGE), and the proteins were transferred onto polyvinylidene difluoride (PVDF) membranes. After the membranes were washed with PBS four times, they were incubated in blocking solution consisting of $5 \%$ skimmed milk powder in Tris-buffered saline with Tween 20 (TBS-T) for $60 \mathrm{~min}$. The membranes were then incubated with rabbit anti-human bFGF (diluted 1:3000, Santa Cruz Biotechnology), rabbit anti-rat VEGF (diluted 1:1000; Santa Cruz Biotechnology), or rabbit anti-rat $\beta$-actin antibodies (diluted 1:500; Sigma, USA) for 60 min. After washing with PBS 4 times, the membranes were incubated with horseradish peroxidase-conjugated goat anti-rabbit secondary antibodies (diluted 1:1000; Santa Cruz Biotechnology) at room temperature for $60 \mathrm{~min}$. After washing in PBS, visualization was performed with alkaline phosphatase (Santa Cruz Biotechnology). The ImageJ analysis software (National Institutes of Health, USA) was used to detect the absorbance of VEGF bands, which were normalized to values obtained for actin to determine relative VEGF expression.

\section{Statistical analysis}

Continuous variables are reported as means $\pm S D$. Independent-sample $t$-tests were performed to compare $\mathrm{GFP}^{+}$and $\mathrm{GFP}^{+} \mathrm{CD}_{3}{ }^{+}$cell counts and the ratios in the GFP-MSC and hbFGF-MSC groups. ANOVA and Bonferroni post hoc tests were performed to compare the differences among the four treatment groups in VEGF vs actin and capillaries vs fibers (as ratios) and capillary counts. Two-sided $P$ values less than 0.05 were considered to be significant. The SPSS 17.0 statistics software (SPSS Inc., USA) was used for the statistical analyses.

\section{Results}

\section{MSC surface marker expression}

The surface marker expression of P3 MSCs was examined using flow cytometry. As shown in Figure 1, the proportions of cells positive for the hematopoietic markers CD11b/c and CD34 (19) were 15.9 and 2.08\%, respectively (Figure $1 \mathrm{~A}$ and $\mathrm{B})$. Conversely, the proportion of cells expressing the MSC markers CD44 and CD90 (20) was 99.9 and $97.7 \%$, respectively (Figure $1 \mathrm{C}$ and D). This surface marker expression profile, in addition to the cellular 

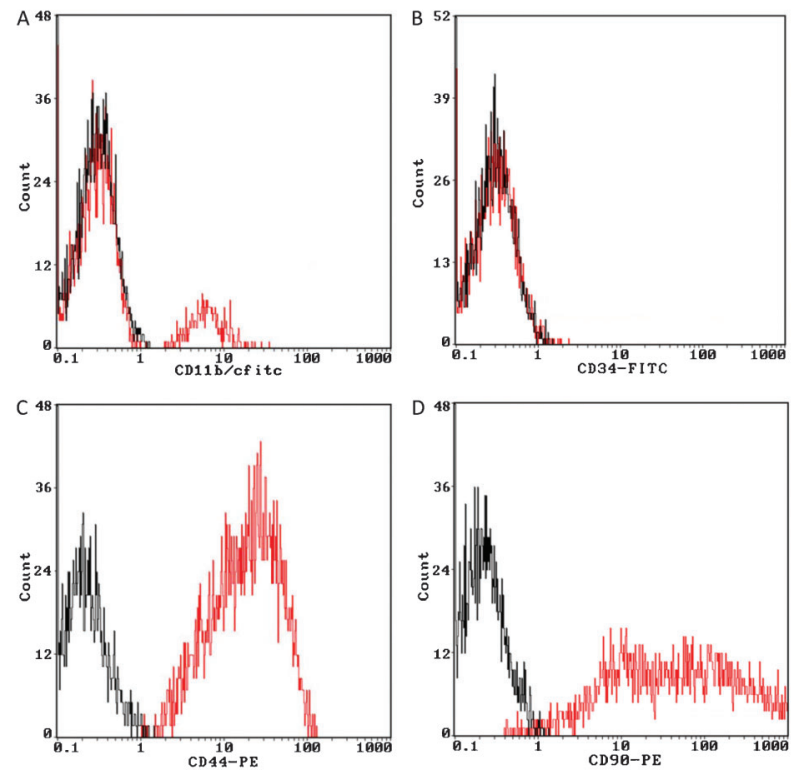

Figure 1. Surface marker expression of isolated rat MSCs (P3). The expression of CD11b/c $(A)$, CD34 $(B)$, CD44 (C), and CD90 $(D)$ was determined using flow cytometry (red lines). Their respective negative controls were also included (black lines).

morphology, confirmed the identity of isolated cells as MSCs. The corresponding negative controls were also included (Figure 1A-D).

\section{Effects of hbFGF-MSCs on VEGF expression in ischemic muscle}

As shown in Figure 2A, hbFGF expression was only observed in animals in the hbFGF-MSC group. In addition, although VEGF expression was observed in the skeletal muscle of animals of all groups, significant differences in relative VEGF expression were observed among the groups (Figure 2B). Specifically, significantly increased VEGF expression was observed in all MSC-treated animals compared to the PBS control group $(\mathrm{P}<0.001)$. Furthermore, the highest VEGF expression was observed in the hbFGF-MSC group, which was significantly higher than in the GFP-MSC group (0.87 vs $0.68 ; \mathrm{P}=0.008)$.

\section{Survival and differentiation of transplanted MSCs}

To assess the differentiation of MSCs that did not express CD31 or Factor VIII (data not shown) into vascular endothelial cells, the coexpression of CD31 and GFP in the gastrocnemius was determined by immunofluorescence analysis 2 weeks after cell transplantation. As shown in Figure 3A, low CD31 expression was observed in the MSC group; however, CD31 expression was increased in both the GFP-MSC and hbFGF-MSC groups. In addition, a fraction of cells was both GFP + and CD31 +, suggesting that differentiation of MSCs into vascular endothelial cells had occurred in these groups. Analysis of the immunofluorescence data
A
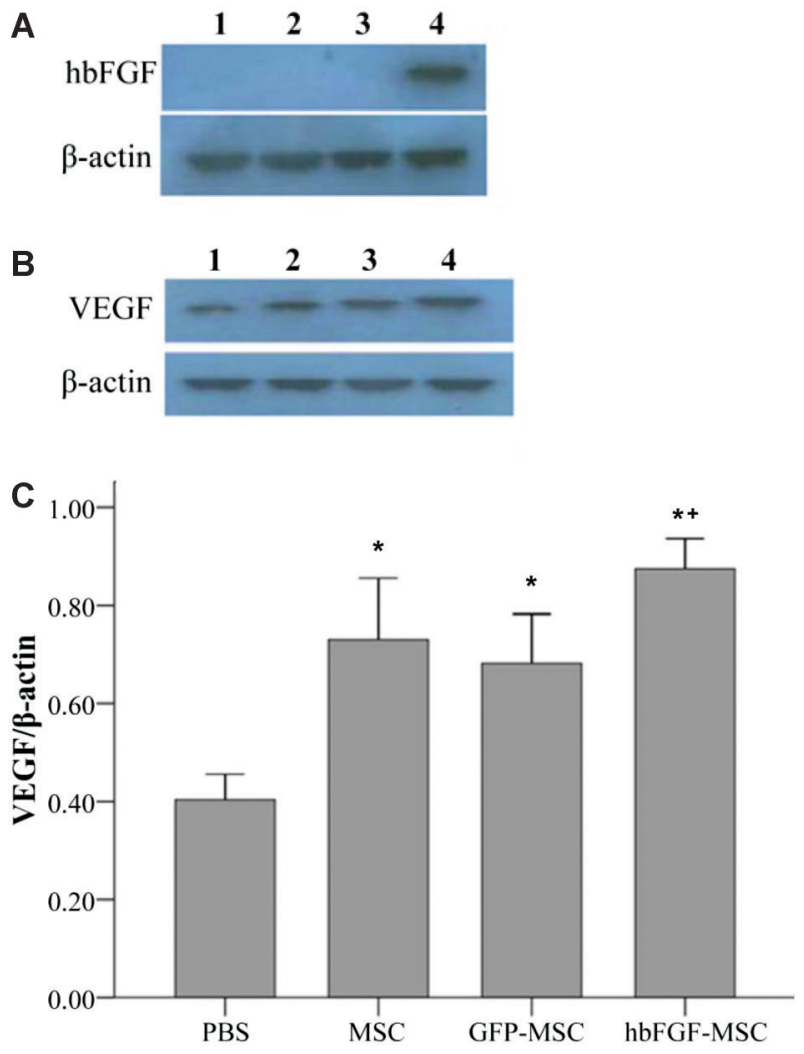

Figure 2. Overexpression of human basic fibroblast growth factor (hbFGF) by mesenchymal stem cells (MSCs) in a rat hind limb ischemia model. $A$, hbFGF and $B$, vascular endothelial growth factor (VEGF) protein expression was determined by Western blot analysis. 1, PBS group $(n=6) ; 2$, MSC group $(n=6) ; 3$, green fluorescent protein (GFP)-MSC group $(n=6) ; 4$, hbFGF-MSC group $(n=6)$. $C$, Relative VEGF expression was determined after normalization to $\beta$-actin. ${ }^{*} P<0.05$, compared to PBS group; ${ }^{+} \mathrm{P}<0.05$, compared to GFP-MSC group ( $\mathrm{n}=6$ for each group) (ANOVA with Bonferroni post hoc tests).

revealed that the number of GFP + cells in the hbFGF-MSC group was significantly higher than in the GFP-MSC group (26.83 vs 15.50, $\mathrm{P}<0.001$; Figure $3 \mathrm{~B}$ ). In addition, the number of GFP + CD31 + cells was also significantly higher in the hbFGF-MSC group compared to the GFP-MSC group (14.33 vs 6.83, $\mathrm{P}<0.001$; Figure $3 C$ ). However, as shown in Figure 3D, no significant difference was observed between the GFP-MSC and hbFGF-MSC groups in the ratio of GFP + CD31 + to GFP + cells, indicating that the differentiation rate did not differ between these two treatment groups.

\section{Effects of hbFGF-MSCs on vasculogenesis}

The in vivo angiogenic potential of hbFGF-MSCs was then determined. The number of microvessels was determined immunohistochemically by analysis of the endothelial cell marker vWF. Representative images of immunohistochemical vWF staining in each of the 4 groups 
$\mathbf{A}$
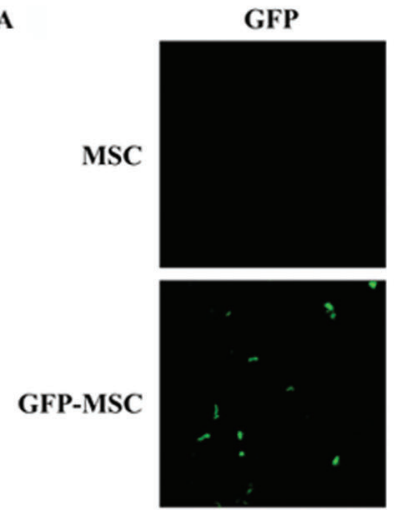

hbFGF-MSC

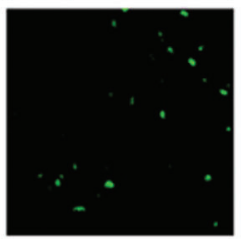

CD31
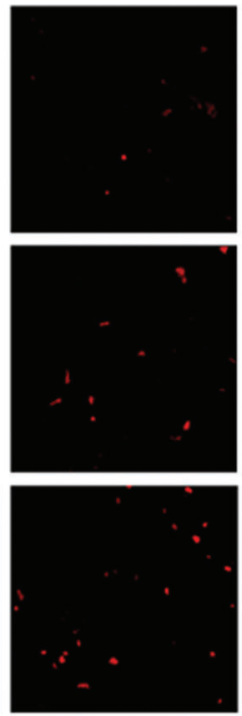
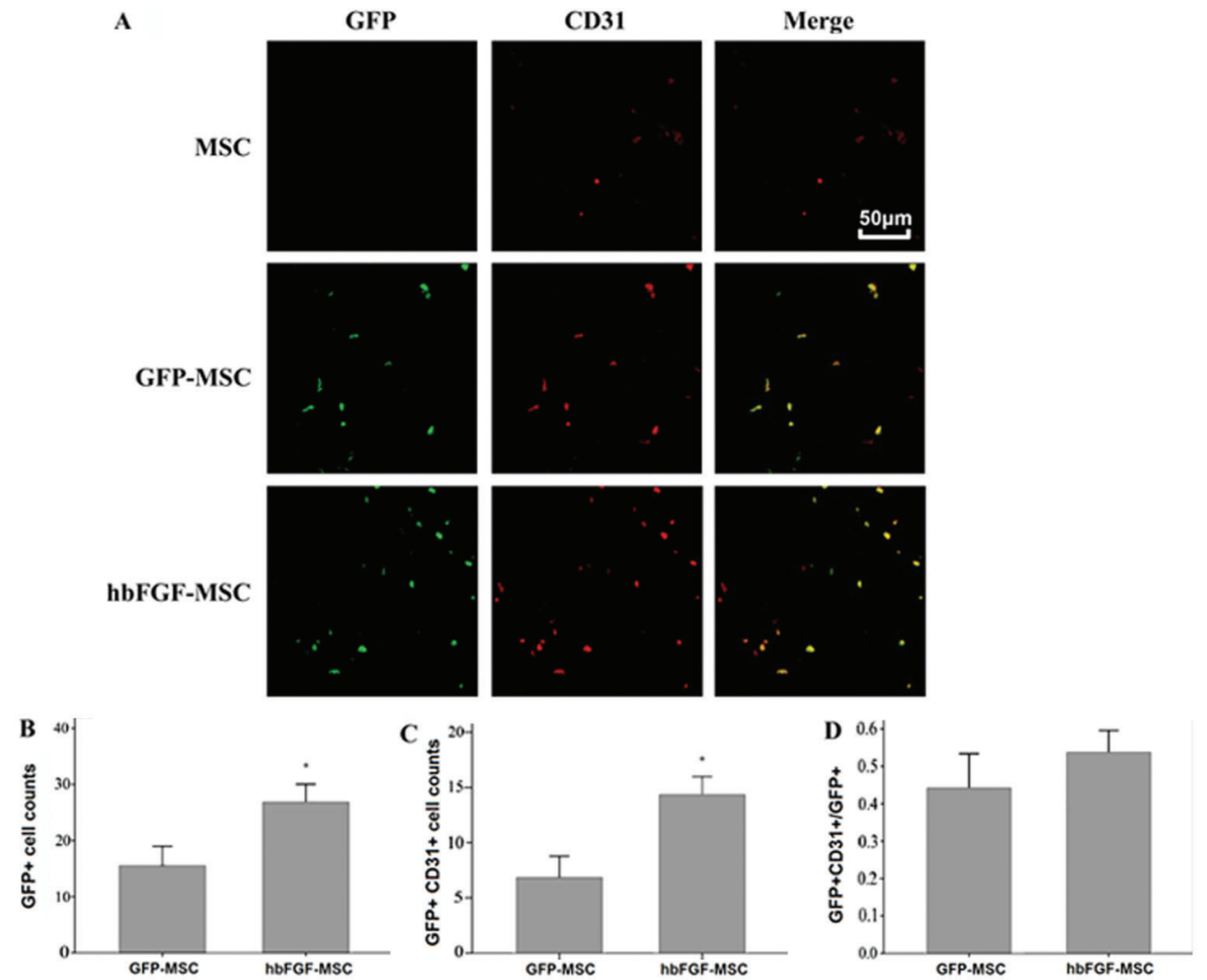

Figure 3. Survival and differentiation of transplanted mesenchymal stem cells (MSCs) in an in vivo hind limb ischemia model. A, Representative images of green fluorescent protein (GFP) (left panels, green) and CD31 (middle panels, red) was determined by immunofluorescence analysis of the gastrocnemius muscle from the MSC, human basic fibroblast growth factor (hbFGF)-MSC, and GFP-MSC groups. Merged images (right panels) identified GFP +CD31+ cells as yellow fluorescence. $B$, MSC survival was determined by counting the number of GFP + cells in the hbFGF-MSC and GFP-MSCs groups ( $n=6 / g r o u p)$. C, MSC differentiation was determined by counting the number of GFP + CD31 + cells in the hbFGF-MSC and GFP-MSC groups $(\mathrm{n}=6 / \mathrm{group})$. $D$, The ratio of GFP + CD31 + cells to GFP + cells represents the relative differentiation rate in the hbFGF-MSC and GFP-MSC groups ( $n=6 / g r o u p)$. ${ }^{*} \mathrm{P}<0.05$, compared to the GFP-MSC group (independent-sample $t$-tests).

are shown in Figure 4A-D. Compared to the PBS control group, the ratio of capillary to fiber counts was significantly higher in the MSC, GFP-MSC, and hbFGF-MSC groups $(P<0.001)$. However, the highest capillary to fiber ratio was observed in the hbFGF-MSC group (0.70 vs 0.41 in the MSC group and 0.42 in the GFP-MSC group, both $\mathrm{P}<0.001$; Figure 4E). Finally, as shown in Figure 4F, capillary counts (microvessel density, MVD) were significantly higher in the GFP-MSC and hbFGF-MSC groups compared to the PBS control group (29.67 and 52.83 vs 8.5 , both $\mathrm{P}<0.001$ ). The capillary counts were significantly higher in the hbFGF-MSC group than in the other groups $(\mathrm{P}<0.001)$.

\section{Discussion}

Inducing angiogenesis is a relatively new therapeutic strategy for the treatment of ischemia. Given the role of hbFGF in inducing angiogenesis, as well as stem cell survival and proliferation (16,22), the effects of bone marrow-derived MSCs overexpressing hbFGF on angiogenesis were analyzed in an in vivo ischemia model. In all of the treatment groups containing MSCs, significantly greater capillary-to-muscle fiber ratios were observed, which is similar to previous reports on the therapeutic potential of MSCs in this model $(7,8)$. Compared to GFPMSCs, a greater number of hbFGF-MSCs survived and differentiated into vascular endothelial cells; however, the differentiation rate was the same. In addition, hbFGFMSCs induced the greatest VEGF expression and MVD.

Various methods of gene transfer have been employed for therapeutic angiogenesis. Administration of recombinant angiogenic factors or direct injection of plasmid DNA into skeletal muscle has been undertaken (6). In addition, stable protein expression of hbFGF was observed in MSCs after transduction of an adeno-associated virus vector (23). In this study, MSCs were transduced with lentiviruses expressing hbFGF, and stable expression was observed at 

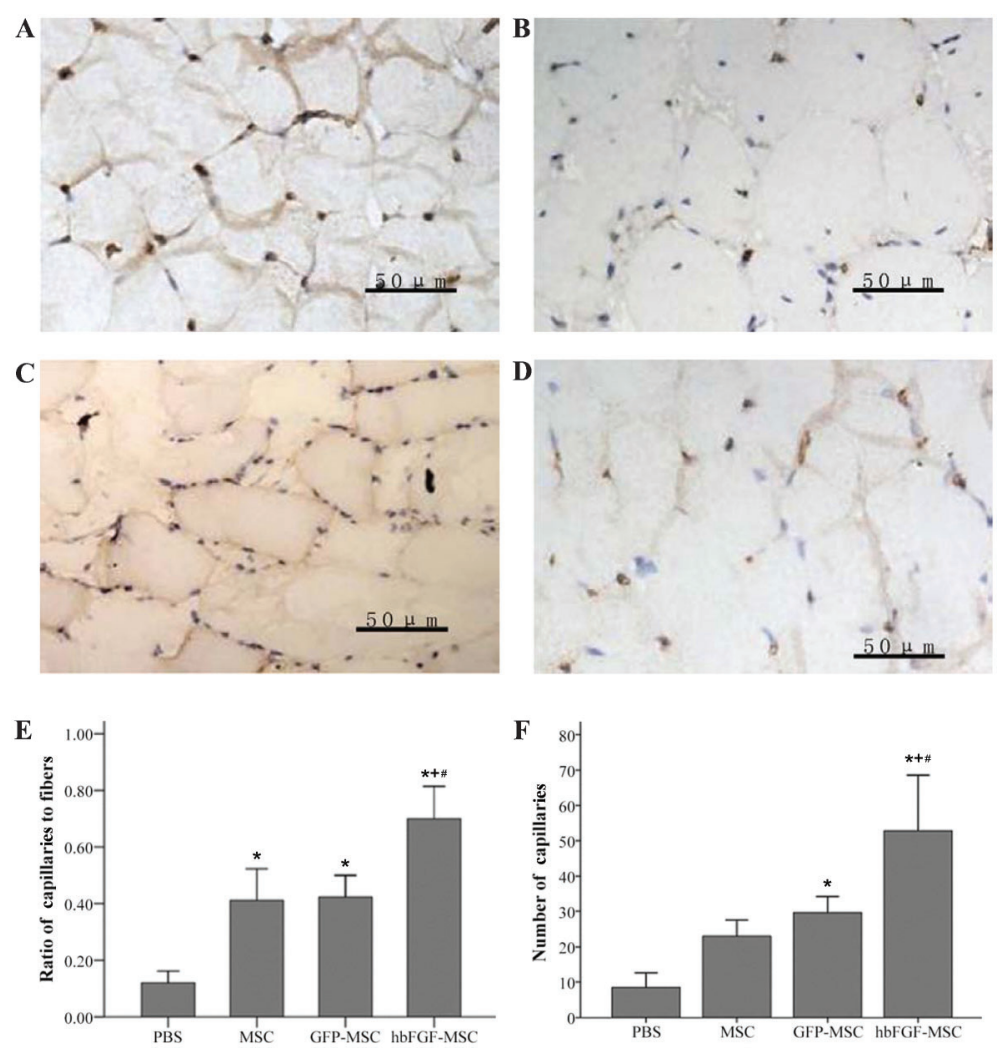

Figure 4. Overexpressing human basic fibroblast growth factor (hbFGF) in mesenchymal stem cells (MSCs) stimulates angiogenesis in a rat hind limb ischemia model. $A-D$, Representative images of sections of ischemic gastrocnemius muscles from the phosphate-buffered saline (PBS) control $(A)$, MSC $(B)$, green fluorescent protein (GFP)-MSC (C), and hbFGF-MSC (D) groups immunostained with anti-von Willebrand factor (vWF) antibodies. After counting the VWFpositive cells (microvessels) and muscle fibers, the ratio of microvessels to muscle fibers $(E)$ and microvessel density $(F)$ were determined. ${ }^{*} \mathrm{P}<0.05$, compared to the PBS group; ${ }^{+} \mathrm{P}<0.05$, compared to the MSC group; ${ }^{\#} \mathrm{P}<0.05$, compared to the GFP-MSC group ( $n=6$ for each group) (one-way ANOVA with Bonferroni post hoc tests).
2 weeks post-transplantation. Further long-term studies will be undertaken to confirm the continued skeletal-muscle expression of hbFGF. In addition to increased hbFGF, hbFGF-MSCs also induced in vivo VEGF expression. However, the exact mechanism by which overexpression of hbFGF in MSCs also increased VEGF expression remains to be determined. It may be an indirect effect of an increased number of MSCs differentiating into endothelial cells. Alternatively, hbFGF expression may directly induce VEGF expression, as observed in human aortic smooth muscle cells (24). Further studies will determine the mechanism responsible for increased skeletal muscle VEGF expression in hbFGF-MSC transplantation.

Although stem cell transplantation has been used to stimulate vasculogenesis with favorable efficacy, the survival rate of stem cells remains low, which negatively impacts their therapeutic efficacy. In the present study, increased MSC survival was observed in those overexpressing hbFGF, which is consistent with previous studies reporting that hbFGF increased survival rate of transplanted stem cells (16), as well as proliferation of stem cells (22). In addition, hbFGF overexpression by MSCs increased the number of cells that differentiated into endothelial cells, but not the relative differentiation rate, suggesting the increased number of GFP + CD31 + cells was due to increased survival and/or proliferation but not increased differentiation capacity. However, the mechanism by which hbFGF influences survival was not determined. bFGF induced both vascular endothelial cell proliferation (25) and endothelial cell migration by inducing the secretion of cathepsin $L$ by skeletal muscle cells (26). In addition, bFGF can simultaneously promote the growth of capillaries and small arteries (27). Furthermore, bFGF can upregulate the expression of the platelet-derived growth factor (PDGF) receptor; therefore, bFGF and PDGF may exert synergistic effects to promote vasculogenesis (28). Further studies will determine the mechanism by which hbFGF expression induces MSCmediated endothelial cell differentiation. Furthermore, additional studies will analyze the effect of coexpression of hbFGF and its coreceptor, syndecan-4, in MSCs, as this combination alone can stimulate neovascularization and increase perfusion in rats with hind limb ischemia (29).

In the present study, both GFP- and hbFGF-expressing MSCs differentiated into endothelial cells in vivo. This differentiation capacity is thought to be the mechanism by which cell therapy restores cardiac function (30), improves blood flow, and reduces necrosis in ischemia $(13,31)$. Specifically, MSCs have been shown to differentiate into cardiomyocytes, vascular smooth muscle cells, and endothelial cells in ischemic cardiomyopathy (30). Furthermore, in an in vivo stroke model, transplantation of brain-derived neurotrophic factor-differentiated, MSCs improved motor function to a greater degree than transplantation of undifferentiated MSCs (32). However, 
the improved blood perfusion in ischemic hind limbs after transplantation of in vitro-differentiated endothelial cells suggests that this differentiation pathway may be necessary, and possibly sufficient, for angiogenic therapy in ischemia (31).

Induction of a microenvironment conducive to stem-cell differentiation and engraftment likely depends on paracrine effects (33) that promote vascularization (e.g., interleukin [IL]-4, IL-13, angiogenin, and VEGF), extracellular matrix production (e.g., PDGF-BB), and tissue remodeling (e.g., matrix metalloproteinases) (13). In addition, differential paracrine factor expression is thought to be responsible for the varying restorative capacity of different stem cells in ischemic diseases (33). That is consistent with the results of the present study, in which greater VEGF production was observed with hbFGF-MSCs than with GFP-MSCs. Further studies will assess the mechanism by which hbFGF-MSCs differentiate into endothelial cells, as well as the engraftment capacity of these cells.

In addition to inducing endothelial differentiation, hbFGF-expressing MSCs significantly increased the MVD by nearly 2 -fold compared to the GFP-MSC group, which is similar to the results of previous studies $(14,15)$. Injection of bFGF-expressing adenovirus into the cerebral ventricle (14) or injection of fibroblasts infected with bFGF-expressing adenovirus into ischemic muscles (15) has been shown to increase the local expression of bFGF, as well as the MVD and number of lateral branches. Furthermore, in a cranial defect model, Chen et al. (23) reported enhanced angiogenesis after treatment with poly-DL-lactide/hydroxyapatite in combination with bFGF-expressing MSCs.

In addition to the direct effect of cytokines, stem cell transplantation may also directly promote vasculogenesis (34). Resident stem cells synthesize VEGF, which then activates vascular endothelial cells via paracrine signaling (35). However, because the extent of VEGF upregulation was not greatly increased in hbFGF-MSCs relative to controls, upregulation of other molecules that affect

\section{References}

1. Schumacher B, Pecher P, von Specht BU, Stegmann T. Induction of neoangiogenesis in ischemic myocardium by human growth factors: first clinical results of a new treatment of coronary heart disease. Circulation 1998; 97: 645-650, doi: 10.1161/01.CIR.97.7.645.

2. Sellke FW, Laham RJ, Edelman ER, Pearlman JD, Simons $M$. Therapeutic angiogenesis with basic fibroblast growth factor: technique and early results. Ann Thorac Surg 1998; 65: 1540-1544, doi: 10.1016/S0003-4975(98)00340-3.

3. Aoki M, Morishita R, Taniyama Y, Kida I, Moriguchi A, Matsumoto K, et al. Angiogenesis induced by hepatocyte growth factor in non-infarcted myocardium and infarcted myocardium: up-regulation of essential transcription factor for angiogenesis, ets. Gene Ther 2000; 7: 417-427, doi: 10.1038/ sj.gt.3301104.

4. Losordo DW, Vale PR, Symes JF, Dunnington CH, Esakof angiogenesis is likely necessary. Stem cells may also synthesize HGF, tumor necrosis factor, and proteases, which promote vascular endothelial cell proliferation and migration (36). Moreover, stem cells have the potential to differentiate into vascular endothelial cells (37), and therefore have a direct role in capillary network formation (38).

The present study is limited in that we did not determine the effects of the various treatment groups on blood flow. In addition, functional experiments to assess recovery after treatment were not carried out. Further studies will evaluate the effects of hbFGF-MSCs on the physiological status of the ischemic limb, including limb rescue, necrosis, and loss, as well as post-treatment exercise capacity. These studies will also help determine the extent of angiogenesis required to achieve clinical benefits. The small proportion of CD11b + cells is indicative of myeloid cell contamination, which may have been eliminated with further passaging. Although Singh et al. (18) also used P3 MSCs, additional studies will determine if this possible contamination affected the findings. Moreover, although previous studies have successfully used hbFGF in rats $(39,40)$, use of rat bFGF might have resulted in greater effectiveness.

Taken together, the data in this study suggest that transplantation of hbFGF-expressing MSCs promotes angiogenesis by increasing the survival of transplanted cells, which subsequently differentiate into vascular endothelial cells. Therefore, patients with ischemic disease may benefit from therapeutic angiogenesis mediated by a combination of gene therapy (i.e., hbFGF overexpression) with allogenic MSC transplantation.

\section{Acknowledgments}

This research was supported by grants from the Provincial University Program of Science and Technology, Department of Fujian Province (\#2008F5022) and the Natural Science Foundation of Fujian Province (\#C0410023).
DD, Maysky M, et al. Gene therapy for myocardial angiogenesis: initial clinical results with direct myocardial injection of phVEGF165 as sole therapy for myocardial ischemia. Circulation 1998; 98: 2800-2804, doi: 10.1161/01.CIR.98.25. 2800.

5. Makarevich P, Tsokolaeva Z, Shevelev A, Rybalkin I, Shevchenko E, Beloglazova I, et al. Combined transfer of human VEGF165 and HGF genes renders potent angiogenic effect in ischemic skeletal muscle. PLoS One 2012; 7: e38776, doi: 10.1371/journal.pone.0038776.

6. Lu Q, Yao Y, Yao Y, Liu S, Huang Y, Lu S, et al. Angiogenic factor AGGF1 promotes therapeutic angiogenesis in a mouse limb ischemia model. PLoS One 2012; 7: e46998, doi: 10.1371/journal.pone.0046998.

7. Hamano K, Li TS, Kobayashi T, Tanaka N, Kobayashi S, Matsuzaki $\mathrm{M}$, et al. The induction of angiogenesis by the 
implantation of autologous bone marrow cells: a novel and simple therapeutic method. Surgery 2001; 130: 44-54, doi: 10.1067/msy.2001.114762.

8. Ikenaga S, Hamano K, Nishida M, Kobayashi T, Li TS, Kobayashi $\mathrm{S}$, et al. Autologous bone marrow implantation induced angiogenesis and improved deteriorated exercise capacity in a rat ischemic hindlimb model. J Surg Res 2001; 96: 277-283, doi: 10.1006/jsre.2000.6080.

9. Asahara T, Murohara T, Sullivan A, Silver M, van der Zee R, $\mathrm{Li} \mathrm{T}$, et al. Isolation of putative progenitor endothelial cells for angiogenesis. Science 1997; 275: 964-967, doi: 10.1126/ science.275.5302.964

10. Noishiki Y, Tomizawa $Y$, Yamane $Y$, Matsumoto A. Autocrine angiogenic vascular prosthesis with bone marrow transplantation. Nat Med 1996; 2: 90-93, doi: 10.1038/nm0196-90.

11. Gutierrez-Fernandez M, Rodriguez-Frutos B, RamosCejudo J, Teresa Vallejo-Cremades M, Fuentes B, Cerdan $S$, et al. Effects of intravenous administration of allogenic bone marrow- and adipose tissue-derived mesenchymal stem cells on functional recovery and brain repair markers in experimental ischemic stroke. Stem Cell Res Ther 2013; 4: 11, doi: $10.1186 /$ scrt159.

12. Madonna R, Taylor DA, Geng YJ, De Caterina R, Shelat H, Perin EC, et al. Transplantation of mesenchymal cells rejuvenated by the overexpression of telomerase and myocardin promotes revascularization and tissue repair in a murine model of hindlimb ischemia. Circ Res 2013; 113: 902-914, doi: 10.1161/CIRCRESAHA.113.301690.

13. Whiteley J, Bielecki R, Li M, Chua S, Ward MR, Yamanaka N, et al. An expanded population of CD34 + cells from frozen banked umbilical cord blood demonstrate tissue repair mechanisms of mesenchymal stromal cells and circulating angiogenic cells in an ischemic hind limb model. Stem Cell Rev 2014; 10: 338-350, doi: 10.1007/s12015-014-9496-1.

14. Yukawa H, Takahashi JC, Miyatake SI, Saiki M, Matsuoka $\mathrm{N}$, Akimoto $\mathrm{M}$, et al. Adenoviral gene transfer of basic fibroblast growth factor promotes angiogenesis in rat brain. Gene Ther 2000; 7: 942-949, doi: 10.1038/sj.gt.3301182.

15. Ohara N, Koyama H, Miyata T, Hamada H, Miyatake SI, Akimoto $\mathrm{M}$, et al. Adenovirus-mediated ex vivo gene transfer of basic fibroblast growth factor promotes collateral development in a rabbit model of hind limb ischemia. Gene Ther 2001; 8: 837-845, doi: 10.1038/sj.gt.3301475.

16. Bhang SH, Lee TJ, La WG, Kim DI, Kim BS. Delivery of fibroblast growth factor 2 enhances the viability of cord blood-derived mesenchymal stem cells transplanted to ischemic limbs. J Biosci Bioeng 2011; 111: 584-589, doi: 10.1016/j.jbiosc.2011.01.003.

17. Zhang JC, Wu MX, Wang YL, Guo PF, Liu XQ. Transfection and expression of basic fibroblast growth factor gene mediated by lentivirus vector into rat mesenchymal stem cells. Chin J Exp Surg 2010; 27: 1436-1438.

18. Singh SP, Tripathy NK, Nityanand S. Comparison of phenotypic markers and neural differentiation potential of multipotent adult progenitor cells and mesenchymal stem cells. World J Stem Cells 2013; 5: 53-60, doi: 10.4252/wjsc. v5.i2.53.

19. Soulas C, Arrighi JF, Saeland S, Chapuis B, Kindler V. Human CD34 + CD11b- cord blood stem cells generate in vitro a a CD34- CD11b + subset that is enriched in langerin + Langerhans dendritic cell precursors. Exp Hematol
2006; 34: 1471-1479, doi: 10.1016/j.exphem.2006.06.011.

20. Tsai MJ, Tsai SK, Hu BR, Liou DY, Huang SL, Huang MC, et al. Recovery of neurological function of ischemic stroke by application of conditioned medium of bone marrow mesenchymal stem cells derived from normal and cerebral ischemia rats. J Biomed Sci 2014; 21: 5, doi: 10.1186/14230127-21-5.

21. Zhang JC, Wang YL, Wu MX, Guo PF. Construction and identification of the recombinant lentiviral vector expressing human bFGF gene. Chin J Exp Surg 2010; 27: 580-582.

22. Takagi M, Nakamura T, Matsuda C, Hattori T, Wakitani S, Yoshida T. In vitro proliferation of human bone marrow mesenchymal stem cells employing donor serum and basic fibroblast growth factor. Cytotechnology 2003; 43: 89-96, doi: 10.1023/B:CYTO.0000039911.46200.61

23. Chen M, Song K, Rao N, Huang M, Huang Z, Cao Y. Effect of bone marrow mesenchymal stem cells transfected with rAAV2-bFGF on early angiogenesis of calvarial defects in rats. J Huazhong Univ Sci Technolog Med Sci 2010; 30: 519-524, doi: 10.1007/s11596-010-0561-1.

24. Belgore F, Lip GY, Blann AD. Basic fibrobrast growth factor induces the secretion of vascular endothelial growth factor by human aortic smooth muscle cells but not by endothelial cells. Eur J Clin Invest 2003; 33: 833-839, doi: 10.1046/ j.1365-2362.2003.01223.x.

25. Watanabe S, Morisaki N, Tezuka M, Fukuda K, Ueda S, Koyama $\mathrm{N}$, et al. Cultured retinal pericytes stimulate in vitro angiogenesis of endothelial cells through secretion of a fibroblast growth factor-like molecule. Atherosclerosis 1997; 130: 101-107, doi: 10.1016/S0021-9150(96)06050-9.

26. Chung JH, Im EK, Jin TW, Lee SM, Kim SH, Choi EY, et al. Cathepsin $L$ derived from skeletal muscle cells transfected with bFGF promotes endothelial cell migration. Exp Mol Med 2011; 43: 179-188, doi: 10.3858/emm.2011.43.4.022.

27. Hao X, Mansson-Broberg A, Gustafsson T, Grinnemo KH, Blomberg $P$, Siddiqui AJ, et al. Angiogenic effects of dual gene transfer of bFGF and PDGF-BB after myocardial infarction. Biochem Biophys Res Commun 2004; 315: 10581063, doi: 10.1016/j.bbrc.2004.01.165.

28. Cao R, Brakenhielm E, Pawliuk R, Wariaro D, Post MJ, Wahlberg $\mathrm{E}$, et al. Angiogenic synergism, vascular stability and improvement of hind-limb ischemia by a combination of PDGF-BB and FGF-2. Nat Med 2003; 9: 604-613, doi: $10.1038 / \mathrm{nm} 848$.

29. Jang E, Albadawi H, Watkins MT, Edelman ER, Baker AB. Syndecan-4 proteoliposomes enhance fibroblast growth factor-2 (FGF-2)-induced proliferation, migration, and neovascularization of ischemic muscle. Proc Natl Acad Sci U S A 2012; 109: 1679-1684, doi: 10.1073/pnas.1117885109.

30. Quevedo HC, Hatzistergos KE, Oskouei BN, Feigenbaum GS, Rodriguez JE, Valdes D, et al. Allogeneic mesenchymal stem cells restore cardiac function in chronic ischemic cardiomyopathy via trilineage differentiating capacity. Proc Natl Acad Sci U S A 2009; 106: 14022-14027, doi: 10.1073/ pnas.0903201106.

31. Lai WH, Ho JC, Chan YC, Ng JH, Au KW, Wong LY, et al. Attenuation of hind-limb ischemia in mice with endothelial-like cells derived from different sources of human stem cells. PLoS One 2013; 8: e57876, doi: 10.1371/journal.pone.0057876.

32. Huang W, Mo X, Qin C, Zheng J, Liang Z, Zhang C. Transplantation of differentiated bone marrow stromal cells 
promotes motor functional recovery in rats with stroke. Neurol Res 2013; 35: 320-328, doi: 10.1179/1743132812Y. 0000000151.

33. Hsieh JY, Wang HW, Chang SJ, Liao KH, Lee IH, Lin WS, et al. Mesenchymal stem cells from human umbilical cord express preferentially secreted factors related to neuroprotection, neurogenesis, and angiogenesis. PLoS One 2013; 8: e72604, doi: 10.1371/journal.pone.0072604.

34. Shintani S, Murohara T, Ikeda H, Ueno T, Sasaki K, Duan J, et al. Augmentation of postnatal neovascularization with autologous bone marrow transplantation. Circulation 2001; 103: 897-903, doi: 10.1161/01.CIR.103.6.897.

35. Salvolini E, Orciani M, Vignini A, Mattioli-Belmonte $M$, Mazzanti L, Di Primio R. Skin-derived mesenchymal stem cells (S-MSCs) induce endothelial cell activation by paracrine mechanisms. Exp Dermatol 2010; 19: 848-850, doi: 10.1111/j.1600-0625.2010.01104.x.

36. Kachgal S, Putnam AJ. Mesenchymal stem cells from adipose and bone marrow promote angiogenesis via distinct cytokine and protease expression mechanisms. Angiogenesis 2011;
14: 47-59, doi: 10.1007/s10456-010-9194-9.

37. Tang J, Xie Q, Pan G, Wang J, Wang M. Mesenchymal stem cells participate in angiogenesis and improve heart function in rat model of myocardial ischemia with reperfusion. Eur $J$ Cardiothorac Surg 2006; 30: 353-361, doi: 10.1016/j.ejcts. 2006.02.070

38. Li TS, Hamano K, Nishida M, Hayashi M, Ito H, Mikamo A, et al. CD117+ stem cells play a key role in therapeutic angiogenesis induced by bone marrow cell implantation. Am J Physiol Heart Circ Physiol 2003; 285: H931-H937.

39. Nakae M, Kamiya H, Naruse K, Horio N, Ito Y, Mizubayashi $R$, et al. Effects of basic fibroblast growth factor on experimental diabetic neuropathy in rats. Diabetes 2006; 55: 1470-1477, doi: 10.2337/db05-1160.

40. Yao CC, Yao P, Wu H, Zha ZG. Absorbable collagen sponge combined with recombinant human basic fibroblast growth factor promotes nerve regeneration in rat sciatic nerve. J Mater Sci Mater Med 2007; 18: 1969-1972, doi: 10.1007/s10856-007-3093-7. 